\begin{tabular}{|c|c|}
\hline Title & Modulation and Characterization of the Double Network Hydrogel Surface-Bulk Transition \\
\hline Author(s) & $\begin{array}{l}\text { Frauenlob, Martin; King, Daniel R.; Guo, Honglei; I shihara, Seiichiro; Tsuda, Masumi; Kurokawa, Takayuki; Haga, } \\
\text { Hisashi; Tanaka, Shinya; Gong, Jian Ping }\end{array}$ \\
\hline Citation & $\begin{array}{l}\text { Macromolecules, 52(17), 6704-6713 } \\
\text { https://doi.org/10.1021/acs.macromol.9b01399 }\end{array}$ \\
\hline Issue Date & $2019-09-10$ \\
\hline Doc URL & http:/hdl.handle.net/2115/79235 \\
\hline Rights & $\begin{array}{l}\text { This document is the A ccepted Manuscript version of a Published Work that appeared in final form in Macromolecules, } \\
\text { copyright } \odot \text { A merican Chemical Society after peer review and technical editing by the publisher. To access the final } \\
\text { edited and published work see https://doi.org/10.1021/acs.macromol.9b01399. }\end{array}$ \\
\hline Tyре & article (author version) \\
\hline Additional Information & There are other files related to this item in HUSCAP. Check the above URL. \\
\hline File Information & Macromolecules_52-17_6704-6713.pdf \\
\hline
\end{tabular}

Instructions for use 


\section{Modulation and characterization of the double network hydrogel surface-bulk transition}

Martin Frauenlob ${ }^{1}$, Daniel R. King ${ }^{2,3}$, Honglei Guo ${ }^{2,3}$, Seiichiro Ishihara ${ }^{2,3}$, Masumi Tsuda ${ }^{3,4,5}$, Takayuki Kurokawa ${ }^{2,3}$, Hisashi Haga ${ }^{2,3}$, Shinya Tanaka ${ }^{3,4,5}$ and Jian Ping Gong ${ }^{2,3,5, *}$

${ }^{1}$ Graduate School of Life Science, Hokkaido University, N21W11, Kita-ku, Sapporo, Hokkaido 0010021, Japan

${ }^{2}$ Faculty of Advanced Life Science, Hokkaido University, N21W11, Kita-ku, Sapporo, Hokkaido 0010021, Japan

${ }^{3}$ Global Institution for Collaborative Research and Education (GI-CoRE), Hokkaido University, Hokkaido 001-0021, Japan

${ }^{4}$ Department of Cancer Pathology, Faculty of Medicine, Hokkaido University, N15W7, Kita-ku, Sapporo 060-8638, Japan

${ }^{5}$ Institute for Chemical Reaction Design and Discovery (WPI-ICReDD), Hokkaido University, N21W10, Kita-ku, Sapporo 001-0021 Japan

*Corresponding author

Name / E-mail address / ORCID iD

Martin Frauenlob_martinfrauenlob@sci.hokudai.ac.jp

0000-0001-7460-9378

Daniel R. King

dking@sci.hokudai.ac.jp

0000-0002-1377-5556

Honglei Guo

hlguo@sci.hokudai.ac.jp

0000-0002-1610-8521

Seiichiro Ishihara

sishihara@sci.hokudai.ac.jp

Masumi Tsuda

tsudam@med.hokudai.ac.jp

Takayuki Kurokawa

kurokawa@sci.hokudai.ac.jp

0000-0001-6834-684X

Hisashi Haga

haga@sci.hokudai.ac.jp

Shinya Tanaka

tanaka@med.hokudai.ac.jp

Jian Ping Gong

gong@sci.hokudai.ac.jp

0000-0003-2228-2750 


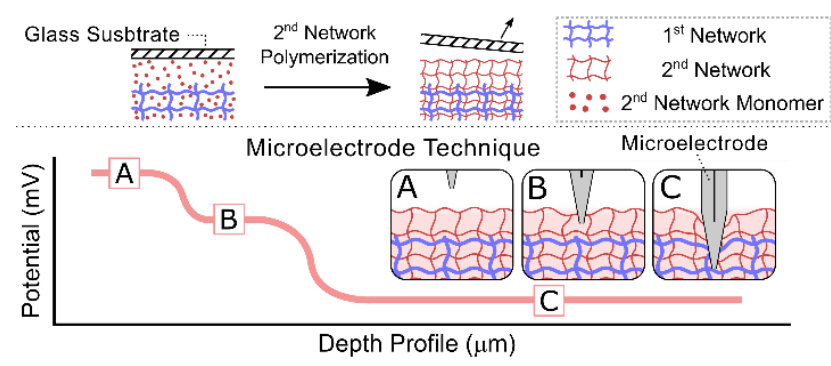

30

\section{Abstract}

The hydrogel chemical structure at the gel-solution interface is important towards practical use, especially in tough double network (DN) hydrogels that have promising applications as structural biomaterials. In this work, we regulate the surface chemical structure of DN hydrogels and the surfacebulk transition by the molding substrate used for the synthesis of the $2^{\text {nd }}$ network. To characterize the surface and bulk structure, we combined ATR-FTIR and a newly developed microelectrode technique that probes the electric potential distribution within a hydrogel. We found that the polymerization on a repulsive substrate leads to the formation of a thin layer of $2^{\text {nd }}$ network on the surface of DN hydrogels, which makes the surface different from the bulk. By controlling the $2^{\text {nd }}$ network polymerization conditions and molding substrate, the surface-bulk transition region can be regulated, so that either only the $2^{\text {nd }}$ network or both networks are present at the DN hydrogel surface. Through these findings we gained a new insight on the structure formation at the DN hydrogel surface, and this leads to easy regulation of the hydrogel surface structure and properties. 
44

\section{Introduction}

The double network (DN) concept gave rise to hydrogels with rubber-like strength and toughness and inspired the development of novel hydrogel materials for potential applications in industrial ${ }^{1-3}$ and medical fields ${ }^{4-6}$. DN hydrogels have structural similarities to living soft matter, and they therefore have received attention for use as cell scaffolds $s^{7,8}$, implants ${ }^{9,10}$ and anti-adhesive membranes ${ }^{11,12}$. For such applications in wet environments, understanding and controlling the surface physicochemical properties of DN hydrogels is crucial.

A typical DN hydrogel owes its high strength and toughness to the contrasting architecture of the two interpenetrating networks: a rigid and brittle $1^{\text {st }}$ network, acting as sacrificial bonds, and a soft and stretchable $2^{\text {nd }}$ network as its interpenetrating counterpart, synthesized in a two-step polymerization approach $^{13-16}$. To obtain the contrasting architecture, a DN hydrogel is usually made from a densely crosslinked polyelectrolyte as the $1^{\text {st }}$ network and a sparsely crosslinked neutral polymer as the $2^{\text {nd }}$ network. Driven by the high osmotic pressure of the counter-ions, the polyelectrolyte network substantially swells in the aqueous precursor solution of the $2^{\text {nd }}$ network, reaching a rigid and brittle state, while the $2^{\text {nd }}$ network, formed in the presence of the first network, can hardly swell due to the constrain of the already highly swollen first network, reaching a soft and stretchable state. Hydrogels made from polyanions such as poly(acrylic acid), poly(sodium p-styrene sulfonate), poly(2-acrylamido2-methyl-propane sulfonic acid) (PAMPS) and PAMPS - sodium salt, have shown many unique properties, including low sliding friction on glass substrates in water, the adsorption of proteins and platelets, known as biofouling, and cell attachment ${ }^{17-19}$. However, after introduction of the $2^{\text {nd }}$ neutral network in such polyelectrolyte hydrogels to create a DN hydrogel, these properties change remarkably, so that cells hardly attach to the DN hydroge ${ }^{20-22}$. Yet, evidence suggests that DN hydrogels from PAMPS and poly(dimethylacrylamide) have therapeutic potential by inducing in vivo articular cartilage regeneration ${ }^{23,24}$, in which the surface physicochemical properties of the DN hydrogels should play an important role in the initial cell adhesion ${ }^{25,26}$ or morphology 27,28 . Understanding and regulating cell-hydrogel interactions are among the most challenging problems in 
bioengineering, and are affected by hydrogel characteristics including charge density ${ }^{29}$, water content $^{30}$, hydrophilicity ${ }^{31}$ and mechanical properties ${ }^{32}$.

In the case of DN hydrogels, it could be argued that cells do not adhere, but do so on the corresponding single network (SN) polyelectrolyte hydrogel, because the concentration of the electrically neutral $2^{\text {nd }}$ network, is already high enough to weaken the surface charge sufficiently ${ }^{33-35}$. Another reason might relate to the two-step synthesis approach and the interactions between the substrate material and the $1^{\text {st }}$ network hydroge $\left.\right|^{36}$. We consider that the surface of the DN hydrogels are sensitive to the two-step polymerization approach and the interaction between the $1^{\text {st }}$ network and the wall of the molding substrate used to synthesize the $2^{\text {nd }}$ network. In the case of a polyelectrolyte hydrogel, at the hydrogelsolution interface, an electric double layer is formed based on the balance of attraction of mobile counter-ions and repellence of mobile co-ions to the immobile ions of the polyelectrolyte network. As a result, there is an electric potential difference between the polyelectrolyte hydrogel and the solution $^{37,38}$. The intensity of the electric potential of the hydrogel in relation to the solution depends on the ionic strength of the solution and the immobile charge density of the hydrogel ${ }^{39}$. Such electric double layer is the origin of repulsion between two like-charged polyelectrolyte hydrogels. When two like-charged surfaces are forced to approach each other, an osmotic repulsion is generated between them to avoid an overlap of the electric double layers of the two surfaces and a liquid film is formed at the interface ${ }^{40}$. Due to the existence of the liquid film as lubricant, two like-charged polyelectrolyte hydrogels show low sliding friction under water ${ }^{41,42}$.

The above results suggest that the surface of a DN hydrogel could be regulated by the molding substrate used for synthesizing the $2^{\text {nd }}$ network. When a polyanion is used as the $1^{\text {st }}$ network and a negatively charged substrate is used as mold, a thin hydrogel layer of the $2^{\text {nd }}$ network should be formed on the surface of the DN hydrogel. In contrast, if a hydrophobic mold is used, to which the $1^{\text {st }}$ network is adhesive, no thin hydrogel layer of the $2^{\text {nd }}$ network will be formed on the DN gel surface. In this work, we synthesized DN hydrogels from PAMPS as the $1^{\text {st }}$ network and polyacrylamide (PAAm) as the $2^{\text {nd }}$ network in a modified two-step synthesis approach. To regulate the surface-bulk transition we used 
substrates of low (silicone coated polyethylene, Si-PET) and high (borosilicate glass substrate) surface energy in the $2^{\text {nd }}$ network polymerization step (Scheme $1 \mathrm{~A}$ ). On the low surface energy substrate, we predict the adhesion of the $1^{\text {st }}$ network to the substrate through hydrophobic interactions resulting in a DN hydrogel (DN/Si-PET) with $1^{\text {st }}$ and $2^{\text {nd }}$ network present at the surface. On the borosilicate glass mold that also carries negative charges on its surface, however, we assume that a PAAm layer forms (DN/Glass) because of the electrostatic repulsion between the PAMPS and the glass. As the thickness of the liquid layer at the repulsive interface is determined by the balance of the ionic osmotic pressure and the normal pressure applied, we tried to regulate the surface layer thickness through applying normal compression to the glass and PAMPS hydrogel in the $2^{\text {nd }}$ network polymerization (DN/Glass-C, Figure S5A).

It is challenging to analyze the heterogeneous DN hydrogel structure, especially if it differs between the surface and the bulk. First, in swollen state, the water molecules and hydrogen bonds absorb a large bandwidth of electromagnetic waves, rendering spectroscopy techniques ineffective ${ }^{43}$. Second, common electric potential characterization methods such as $\zeta$-potential measurements, used to determine hydrogel charges, are not capable of analyzing a structure-related charge difference between the surface and bulk of the hydroge ${ }^{44}$. To overcome these difficulties, we adopted a recently developed method that provides a new perspective on the charge distributions in hydrogels, called the microelectrode technique (Scheme $1 \mathrm{~B})^{45}$. By inserting a microelectrode into the hydrogel and recording the electric signal in accordance to the electrode penetration velocity, an electric potentialdepth profile of the hydrogel is generated, which reveals the charge density distribution inside of the hydrogel. Here in this study we deployed attenuated total reflectance Fourier-transform infrared spectroscopy (ATR FT-IR) and the microelectrode technique to characterize the surface and bulk structure of the DN hydrogels. ATR FT-IR provides surface structure information while the microelectrode technique is a highly sensitive method giving a depth profile with a low spatial resolution of the microelectrode diameter ( $200 \mathrm{~nm})$. Using these two complementary methods, we 
can characterize the surface layer structure and the surface-bulk transition of the hydrogels (Scheme

122 1C).

123 Ultimately, we identified that modulating the $2^{\text {nd }}$ network polymerization condition regulates the

124 surface-bulk transition and allows us to switch-on or switch-off the cell adhesive properties on DN

125 hydrogels. By modifying the two-step DN hydrogel synthesis approach we provide an easily accessible opportunity to design tough hydrogels with functional surface charge transitions, which are useful in cell scaffolding and in vivo applications.
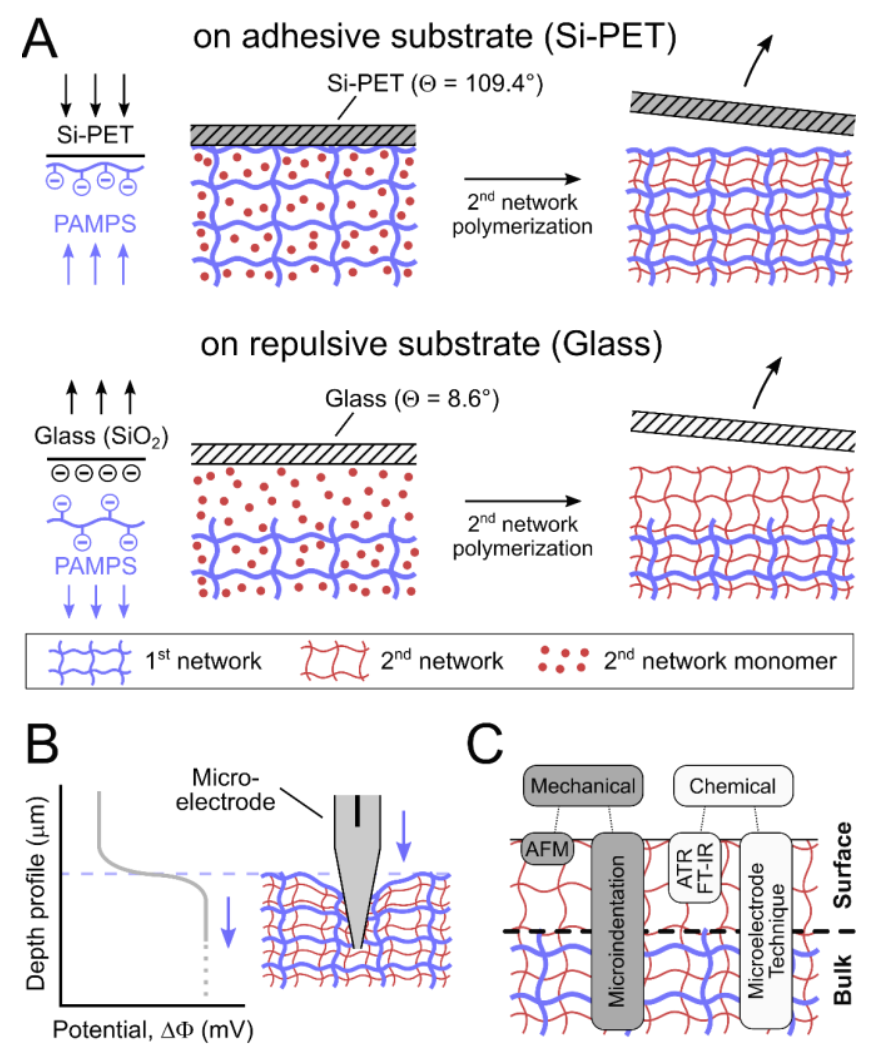

Scheme 1: Strategy to regulate and to analyze the surface-bulk transition of DN hydrogels by using different molds for second network synthesis. (A) DN hydrogels synthesized on the hydrophobic substrate (Si-PET) have surfaces containing both polymer networks due to adhesion of the $1^{\text {st }}$ network on the substrate. DN hydrogels synthesized on the glass substrate are covered with a thin layer of $2^{\text {nd }}$ network polymer due to the osmotic repulsion between the negatively charged glass and the $1^{\text {st }}$ network. (B) The microelectrode technique measures the electric potential of the hydrogel depth profile (blue dotted line represents the hydrogel-solution interface). (C) Summary of the deployed mechanical and chemical characterization methods used to analyze the surface-bulk transition of DN hydrogels. 


\section{Experimental Section}

139

\section{Materials}

The $1^{\text {st }}$ network monomer 2-acrylamido-2-methylpropanesulfonic acid (AMPS) (Toagosei Co., Ltd., Japan) was used as received. The $2^{\text {nd }}$ network monomer acrylamide $(A A m)$, crosslinker $N, N^{\prime}-$ methylenebis(acrylamide) (MBAA) and initiator 2-oxoglutaric acid were used as obtained from Wako Pure Chemical Industries, Ltd., Japan. The molding surfaces for the $2^{\text {nd }}$ network polymerization, such as borosilicate glass substrate (Asahi Glass Co., Ltd., Japan), were heat treated at $500{ }^{\circ} \mathrm{C}$ for 2 hours prior to use and silicone coated polyethylene film (Si-PET), SP-PET-03-38-Bu (Mitsui Chemicals Tohcello. Inc., Japan) was used as purchased. Unless otherwise noticed, all chemicals and solvents used in cell culture were purchased from Sigma-Aldrich Inc, United States. The human mesenchymal stem cell (MSC) line derived from umbilical cord blood (UCB) (JCRB1110 UCB408E6E7TERT-33) was obtained from the Japanese Collection of Research Bioresources Cell Bank (JCRB Cell Bank, Japan).

\section{Surface energy of molding substrates}

To characterize the surface energy of the molding substrates used in the $2^{\text {nd }}$ network polymerization step, the static water contact angles $(\Theta)$ of the glass substrate and the Si-PET film were measured with double distilled water $\left(\mathrm{ddH}_{2} \mathrm{O}\right.$, droplet volume $2 \mu \mathrm{L}$ ) on a Drop Master 300 (Kyowa Interface Science Co., Ltd.). The contact angle images are found in the supplementary information in Figure S1.

\section{Single and double network hydrogel synthesis}

The DN hydrogel synthesis was modified from Gong et.al ${ }^{46}$ and sample codes and formulations are found in Table 1. Briefly, in the first step, aqueous precursor solutions of AMPS monomer $\left(C_{M 1}\right)$, crosslinker MBAA $\left(X_{X 1}\right)$ and initiator 2-oxoglutaric acid $\left(X_{11}\right)$ were poured into glass molds with a spacer thickness of 0.5 to $2 \mathrm{~mm}\left(h_{0}\right)$ and free-radical polymerization was initiated by irradiated of $365 \mathrm{~nm}$ UV light in argon atmosphere ( 2.5 mbar) for 8 hours to obtain $1^{\text {st }}$ network PAMPS hydrogels. This first 
polymerization process was followed by 3-day immersion of the freshly synthesized hydrogels in the $2^{\text {nd }}$ network precursor solution composed of AAm monomer $\left(C_{M 2}\right)$, crosslinker MBAA $\left(X_{X_{2}}\right)$ and initiator 2-oxoglutaric acid $\left(X_{12}\right)$. In the second step, the PAMPS hydrogels soaked in the $2^{\text {nd }}$ network precursor solution, were sandwiched between glass plates (hereafter referred as DN/Glass) or Si-PET film (referred as DN/Si-PET) and compressed by $0.1-0.3 \mathrm{kPa}$. For the DN sample group DN/Glass-C, the PAMPS hydrogels were sandwiched between glass plates and compressed by $50 \mathrm{kPa}$ (Figure S5A). For the thickness gradient DN hydrogels, the PAMPS hydrogel was sandwiched between glass plates in a mold with a gradual increase in spacer thicknesses (Scheme S1). The hydrogel-mold assemblies were incubation in the argon atmosphere for 1 hour, and the $2^{\text {nd }}$ network was polymerized within the PAMPS network by UV irradiation for 8 hours.

Single network (SN) hydrogels corresponding to the $1^{\text {st }}$ or $2^{\text {nd }}$ network were synthesized in a similar manner to the $1^{\text {st }}$ network polymerization of DN hydrogels from aqueous solutions of either AMPS or AAm monomer with various crosslinker densities of MBAA. Prior to hydrogel characterization, the synthesized SN and DN hydrogels were equilibrated in double distilled water $\left(\mathrm{ddH}_{2} \mathrm{O}\right)$ for at least for 7 days.

Table 1: Hydrogel sample codes and formulations

\begin{tabular}{|c|c|c|c|c|c|c|}
\hline \multirow[t]{3}{*}{ Sample code } & \multicolumn{3}{|c|}{ PAMPS - $1^{\text {st }}$ network } & \multicolumn{3}{|c|}{ PAAm - $2^{\text {nd }}$ network } \\
\hline & Monomer & Crosslinker* & Initiator* & Monomer & Crosslinker* & Initiator* \\
\hline & $C_{M l}(\mathrm{M})$ & $X_{X I}(\mathrm{~mol} \%)$ & $X_{I I}(\mathrm{~mol} \%)$ & $C_{M 2}(\mathrm{M})$ & $X_{X 2}(\mathrm{~mol} \%)$ & $X_{I 2}(\mathrm{~mol} \%)$ \\
\hline PAMPS $\left(\mathrm{C}_{\mathrm{M} 1}-X_{X I}-X_{I I}\right)$ & 1 & $1.5,2,3,5,9$ & 1 & - & - & - \\
\hline PAAm $\left(\mathrm{C}_{M 2}-X_{X 2}-X_{I 2}\right)$ & - & - & - & 2 & $0.1-4$ & 0.1 \\
\hline $\mathrm{DN}\left(\mathrm{C}_{M 1}-X_{X 1}-X_{I I} / \mathrm{C}_{M 2}-X_{X 2}-X_{I 2}\right)$ & 1 & $1.5,2,3,5,9$ & 1 & 2 & 0.1 & 0.1 \\
\hline
\end{tabular}

*mol\% of $X_{X 1}, X_{I l}$ and $X_{X 2}, X_{I 2}$ are relative to the amount of $\mathrm{C}_{M I}$ and $C_{M 2}$, respectively.

\section{Hydrogel swelling and polymer weight fraction}

The bulk volumetric swelling degree of PAMPS hydrogels, $Q=\left(h / h_{0}\right)^{3}$, was determined from the thickness of PAMPS hydrogels swollen in double distilled water $\left(\mathrm{ddH}_{2} \mathrm{O}\right)$ or $2^{\text {nd }}$ network precursor solution $(h)$, and that in the as-prepared-state $\left(h_{0}\right)$ after polymerization. The polymer weight fraction, $W_{M 1}$ and $W_{M 2}\left(\right.$ wt\%) of SN hydrogel was determined by the gravimetric wet $(m) /$ dry $\left(m_{0}\right)$ ratio $(\times 100)$ 
of hydrogel equilibrated in $\mathrm{ddH}_{2} \mathrm{O}(\mathrm{m})$, and after vacuum oven drying procedure at $75^{\circ} \mathrm{C}$ and reduced pressure of 1 bar for at least 2 days $\left(m_{0}\right)$.

\section{Polymer surface density via ATR/FT-IR}

To analyze polymer surface density of the $1^{\text {st }}$ and $2^{\text {nd }}$ network at the SN and DN hydrogel surface, attenuated total reflection Fourier transform infrared spectroscopy (ATR/FT-IR) on a FT-IR 6600 spectrometer (Jasco, Japan) with diamond prism was performed. Prior to analysis, hydrogel discs were immersed in $\mathrm{D}_{2} \mathrm{O}$ for $12 \mathrm{~h}$ and hydrogel spectra were measured in full contact with the prism for wavenumbers of $1800-800 \mathrm{~cm}^{-1}$, at $16 x$ accumulation and resolution of $2 \mathrm{~cm}^{-1}$. Absorbance maxima ${ }^{47}$, $\left(A_{\max }\right)$ of the characteristic sulfonic group peak $\left(v_{\mathrm{S}=0}\right)$ of PAMPS at $1042 \mathrm{~cm}^{-1}$, and carbonyl group peak $\left(v_{\mathrm{c}=0}\right)$ for PAAm at $1640 \mathrm{~cm}^{-1}$ were determined using the manufacturers software. Since infrared absorption peak height resembles polymer surface density, a calibration curve that relates IR absorption peak height and polymer weight fraction of $\mathrm{SN}$ hydrogel allows the detection of $1^{\text {st }}$ and $2^{\text {nd }}$ network at the DN hydrogel surface (Table S1, Figure S3, Figure S4). Since the gels slightly swelled in deuterium oxide compared to water, the relative swelling ratio $\left(Q_{r}\right)$ was used as a correction factor for the polymer surface density calibration. The $Q_{r}=\left(h_{H 2 O} / h_{D 2 O}\right)^{3}$ was determined by the hydrogel thickness in $\mathrm{ddH}_{2} \mathrm{O}\left(h_{\mathrm{H} 2 \mathrm{O}}\right)$ relative to thickness in deuterium oxide $\left(h_{D 20}\right)$, (Figure S4). Polymer surface densities were expressed as weight fractions and as PAMPS to PAAm molar concentration ratio, $C_{M 1} / C_{M 2}(\mathrm{M} / \mathrm{M})$.

\section{Depth profiling via microelectrode technique}

The surface layer thickness $(t)$ at which the hydrogel structure transitions from the surface to bulk was evaluated by a microelectrode insertion technique previously described ${ }^{45}$. Here the thickness is based on the displacement of the microelectrode moving at a constant speed and the shift in electric potential $(\Delta \Phi)$ due to the relative change in the local small ion concentration. Equilibrated in $10^{-4} \mathrm{M}$ $\mathrm{NaCl}$ solution, hydrogels were probed with a microelectrode made from a glass capillary with a fiber filament (outer diameter $=180 \mathrm{~nm}$, inner diameter $=128 \mathrm{~nm}$ ) fabricated with a P-1000 capillary puller 
$\left(\Delta \Phi_{S}\right)$ were estimated from the $1^{\text {st }}$ derivative of the electric potential curves and secondly the peak distance measured from the first valley to the last hill of the $2^{\text {nd }}$ derivative was used to estimate the surface layer thickness $(t)$ of hydrogels (Figure S2). The $1^{\text {st }}$ and $2^{\text {nd }}$ derivative curves were processed via smoothing and $y$-axis scaling in Origin (OriginLab Corp.) software. The Debye length of the bath solution $\left(10^{-4} \mathrm{M} \mathrm{NaCl}\right)$ is $30.4 \mathrm{~nm}^{45}$.

\section{Mechanical characterization of hydrogels}

The surface elastic modulus ( $\left.E_{\text {surface }}\right)$ was tested on an atomic force microscope (AFM, Nanowizard 4, Bruker, U.S.) mounted to an inverted microscope (Nikon Eclipse TE 300, Japan) in aqueous conditions at $25^{\circ} \mathrm{C}$. The spring constants of $0.079-0.083 \mathrm{~N} / \mathrm{m}$ were obtained by thermal tuning prior to each $4 \mathrm{x}$ 4 force-distance curve array in force mapping-mode on the SFM (QI ${ }^{\mathrm{TM}}$ - mode) for the used pyramid shaped cantilever (A triangular, MLCT series, Bruker, U.S.). The peak force setpoint was normalized to $0.2 \mathrm{nN}$. The bulk elastic modulus $\left(E_{\text {Bulk }}\right)$ was determined by indentation method using a tensilecompressive mechanical tester (Autograph AG-X, Shimadzu Co., Japan) in air at $25^{\circ} \mathrm{C}$. A sphere-shaped micro-indenter ( $r=250 \mu \mathrm{m}$, material: stainless steel) probed the hydrogel at a deformation velocity of $250 \mu \mathrm{m} / \mathrm{min}$. By applying the Hertzian model ${ }^{48}$ for rigid sphere indenters, with an assumed Poisson's ratio for the hydrogels of 0.5 the elastic modulus $(E)$ was estimated from the AFM and microindentation loading curves.

\section{Hydrogel cross-section structure observation}

The swollen DN hydrogel sample with a surface layer thickness gradient was frozen in liquid $\mathrm{N}_{2}$ and fractured to expose the cross section and freeze-dried (Advantage XL-70, VirTis freeze dryer). After gold sputtering (E-1010, Hitachi, Japan), the structure of the hydrogel cross-section was analyzed on a scanning electron microscope (SEM, JSM-6010LA, JEOL Ltd., Japan). 


\section{In vitro cell culture and morphology}

231 Hydrogel membranes were equilibrated and sterilized in HEPES-buffer and then incubated in culture

232 medium overnight. The Dulbecco's modified Eagle's culture media was supplemented with $10 \%$ fetal

233 bovine serum (Thermo Fisher Sci.), 1\% Penicillin/Streptomycin and 1\% L-glutamine. The mesenchymal

234 stem cells were expanded on polystyrene dishes prior to cell seeding on hydrogel membranes. On day

2350 , trypsinized single-cell suspensions of stem cells were tested by trypan blue dye live/dead exclusion.

236 Cell cultures with a viability over $95 \%$ were seeded onto hydrogel membranes at a concentration of 1

$237 \times 10^{5}$ cells $/ \mathrm{mL}$ and incubated for 3 days at $37^{\circ} \mathrm{C}$ and $5 \% \mathrm{CO}_{2}$ in humidified atmosphere. Cell cultivation

238 was stopped on day 3 and cell morphologies were imaged through phase-contrast microscopy

239 (OLYMPUS - IX73, Japan). 


\section{Results and Discussion}

242 Chemical and mechanical properties of the surface-bulk transition region

243 First, we characterized the surface chemical compositions of DN hydrogels prepared on different

244 substrates using ATR/FT-IR which reveals the chemistry of the topmost surface of the hydrogels.

245 PAMPS has a sulfonic group and PAAm has an amide carbonyl group (Figure 1A) that can be

246 characterized by ATR/FT-IR. On the absorption spectra (Figure 1B), a sharp peak at $1042 \mathrm{~cm}^{-1}$,

247 characteristic for the sulfonic group $\left(v_{\mathrm{s}=0}\right)$ of the PAMPS network, and a strong sharp absorption peak

248 around $1640 \mathrm{~cm}^{-1}$, from the primary amide carbonyl group $\left(v_{\mathrm{c}=0}\right)$ of PAAm, were observed on the

249 spectra for the SN PAMPS (1-3-1) gel and PAAm (2-0.1-0.1) gel, respectively. On the PAMPS hydrogel,

250 a peak at $1640 \mathrm{~cm}^{-1}$ is also observable since the molecular structure of PAMPS contains a primary amide

251 carbonyl group $\left(v_{\mathrm{C}=0}\right)$. However, the signal is much weaker compared to PAAm and therefore it is

252 neglectable. The spectrum of the DN/Si-PET, exhibits both strong absorption peaks at $1042 \mathrm{~cm}^{-1}$ for

253 the sulfonic group and at $1640 \mathrm{~cm}^{-1}$ for the primary amide carbonyl group, while the DN/Glass hydrogel

254 only exhibits absorption at $1640 \mathrm{~cm}^{-1}$ due to the amide carbonyl group. These ATR/FT-IR results

255 indicate that both PAMPS and PAAm are present on the surface of the DN/Si-PET hydrogel while only

256 the $2^{\text {nd }}$ network PAAm is present on the surface of the DN/Glass hydrogel. Based on this analysis, we

257 infer that there is a surface layer of PAAm on the DN/Glass hydrogel. 
A

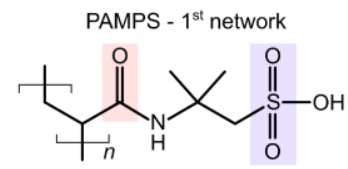

B

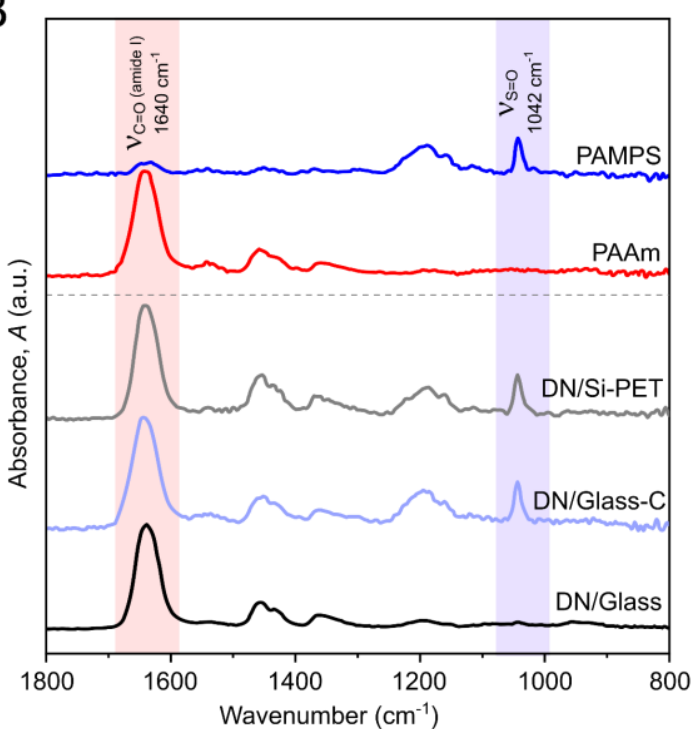

PAAm - $2^{\text {nd }}$ network

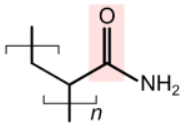

(1)

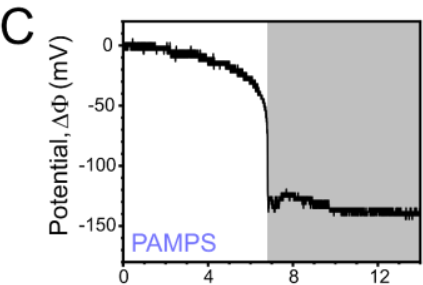

Depth profile $(\mu \mathrm{m})$

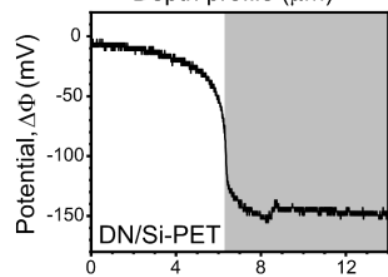

Depth profile $(\mu \mathrm{m})$

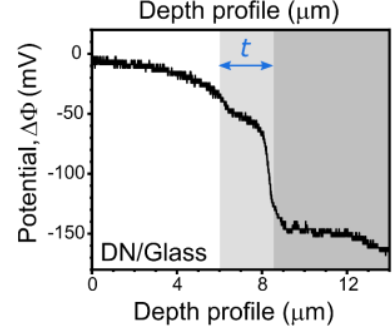

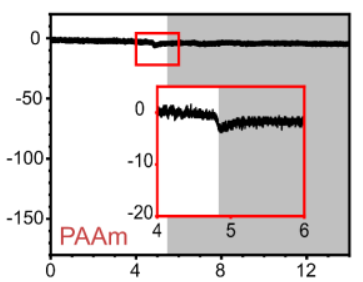

Depth profile $(\mu \mathrm{m})$

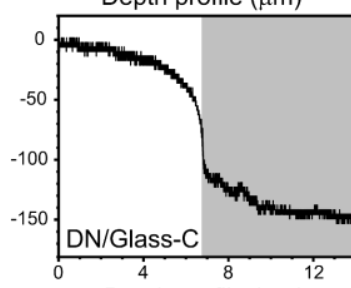

Depth profile $(\mu \mathrm{m})$

- Potential

$\square$ Solution

$\square$ Surface layer

$\square$ Hydrogel bulk

$\stackrel{t}{\longleftrightarrow}$ Surface layer thickness

Figure 1: Analysis of the surface structure of DN hydrogels where the $2^{\text {nd }}$ network was synthesized on different substrates. (A) The molecular structures of the $1^{\text {st }}$ and $2^{\text {nd }}$ network polymers. The red and blue background represent the IR bond absorptions, used to characterize the hydrogels. (B) The ATR/FT-IR absorption spectra and (C) the electric potential depth profiles $(\Delta \Phi)$ of SN PAMPS (1-3-1), SN PAAm (2-0.1-0.1) and corresponding DN hydrogels (1-3-1/2-0.1-0.1) synthesized on Si-PET (DN/SiPET) and glass substrates (DN/Glass), and on glass under compression (DN/Glass-C, Figure S5).

To characterize the thickness of the surface layer on the DN/Glass hydrogel, we probed the depth profile of the electric potential near the surface of the hydrogels by the microelectrode technique. A charged hydrogel generates an electric potential $(\Delta \Phi)$ relative to the bath aqueous solution due to the Donnan effect ${ }^{49}$.

$$
\Delta \Phi=-\frac{2.3 R T}{z F} \log \left(\frac{C_{g}}{C_{s}}\right)
$$

Here, $C_{g}$ and $C_{s}$ are the mobile counter-ion concentrations in the hydrogel and in the bath solution, respectively. $\mathrm{z}$ is the valence of the mobile counter-ion, $\mathrm{F}$ the Faraday constant, $\mathrm{R}$ the gas constant, and $\mathrm{T}$ the absolute temperature. The covalent bound ions on the polymer network guide the mobile counter-ion concentration to keep neutrality in a thermodynamic equilibrium state. As a result, by probing the local electric potential, we can detect the local structure of the hydrogel. For the PAMPS hydrogel, that carries immobile negative charges on the polymer network, the potential shifts to a negative value relative to the bath solution since the hydrogel traps high numbers of positively charged 
mobile ions ( $z$ is positive), while the PAAm hydrogel has almost zero potential since its polymer network carries a negligible amount of charge. Accordingly, we can probe the surface layer thickness of the DN/Glass hydrogels via the electric potential depth profiling.

As shown in Figure 1C, the microelectrode, inserted into a SN PAMPS hydrogel, measures an initial gradual drop of electric potential until the displacement of $6.8 \mu \mathrm{m}$, which is followed by an abrupt drop to a large negative value of $-150 \mathrm{mV}$. The initial potential drop is due to the deformation before the penetration of the electrode into the hydrogel. Like the piezoelectric effect, the compression at the probe tip increases the local polymer charge density and decreases the electric potential. The large potential shift $(\Delta \Phi)$ to $-150 \mathrm{mV}$ indicates that a high amount of $\mathrm{Na}^{+}$ions $(\mathrm{z}=+1)$ are deployed as counterions to neutralize the fixed negative charges of the sulfonic groups of PAMPS. In contrast, only a very small potential shift of $-4 \mathrm{mV}$ is measured for the neutral SN PAAm hydrogel. The potential depth profile of the DN/Si-PET hydrogel, where the $2^{\text {nd }}$ network was synthesized on adhesive Si-PET (contact angle to $\mathrm{ddH}_{2} \mathrm{O} \Theta=109.4 \pm 0.8^{\circ}$, Figure S1), shows an immediate drop of the potential to $-150 \mathrm{mV}$ after the initial drop coming from the compression before electrode penetration, indicating the absence of the surface layer, in good agreement with the ATR/FT-IR result.

In contrast, the depth profiles of the DN/Glass hydrogel (1-3-1/2-0.1-0.1), where the $2^{\text {nd }}$ network was synthesized on repulsive glass (contact angle to $\mathrm{dd} \mathrm{H}_{2} \mathrm{O}, \Theta=8.5 \pm 1.5^{\circ}$, Figure $\mathrm{S} 1$ ), also shows an initial drop of electric potential to a probe displacement of $6.1 \mu \mathrm{m}$, like the SN PAMPS hydrogel before the probe penetration to the hydrogel. Different from the profile of the SN PAMPS hydrogel, a small hump with a change in potential of $-50 \mathrm{mV}$ appears at the displacement of $6.1 \mu \mathrm{m} \sim 8.5 \mu \mathrm{m}$, indicating the presence of a weakly charged surface layer. At a displacement larger than $8.5 \mu \mathrm{m}$, the potential shifts drastically to $-150 \mathrm{mV}$, indicating the transition from the surface layer to the bulk material composition of PAMPS/PAAm because the shift intensity is like that of the corresponding PAMPS SN hydrogel. From the $2^{\text {nd }}$ derivative of the depth profile, a surface layer of thickness $t=2.23 \mu \mathrm{m}$ on the DN/Glass hydrogel was determined (Figure S2). 
The microelectrode results reveal the presence of a surface layer on the DN/Glass hydrogel while no surface layer is formed on the DN/Si-PET hydrogel. These results are consistence with the ATR/FT-IR results. However, the microelectrode method reveals that the surface layer on the DN/Glass hydrogel has a larger electric potential $(-50 \mathrm{mV})$ than that of the corresponding PAAm hydrogel $(-4 \mathrm{mV})$, indicating that the surface layer of the DN/Glass hydrogel is not pure PAAm, which is not consistent with the ATR/FT-IR measurement that show no signal from PAMPS. This discrepancy is due to the relatively poor sensitivity of the ATR/FT-IR. From the potential shift of $-50 \mathrm{mV}$ in relation to the bath solution containing $10^{-4} \mathrm{M} \mathrm{NaCl}$, the concentration of mobile $\mathrm{Na}^{+}$ion in the surface layer of the DN/Glass gel is estimated as $7 \times 10^{-4} \mathrm{M}$ by using Equation 1 . The presence of this excess number of mobile ions in the surface layer of the DN/Glass hydrogel suggests that a very small amount of AMPS monomers, as residue from the $1^{\text {st }}$ network synthesis, of $7 \times 10^{-4} \mathrm{M}$ in concentration, is copolymerized in the $2^{\text {nd }}$ PAAm polymerization to form a copolymer. Such a small amount of AMPS in the surface layer of the DN/Glass could not be detected by the ATR/FT-IR method that has a lower detection limit for PAMPS of $9.4 \times 10^{-4} \mathrm{M}$ (Figure S3).

The PAAm surface layer formation on the DN/Glass hydrogel is presumably caused by the counter ions osmotic repulsion between negatively charged PAMPS hydrogel and the glass substrate during the $2^{\text {nd }}$ network polymerization. To verify the hypothesis, we tried to eliminate the surface layer by applying compression $(50 \mathrm{kPa})$ to the $1^{\text {st }}$ network hydrogel during the synthesis of the $2^{\text {nd }}$ network to counteract the osmotic repulsion (Figure S5A). The ATR/FT-IR spectra show both peaks from PAAm $\left(1640 \mathrm{~cm}^{-1}\right)$ and PAMPS (1042 $\mathrm{cm}^{-1}$ ) (Figure 1B), indicating no surface layer formation on the DN/Glass-C hydrogel. The electric potential depth profile also confirms no surface layer formation. These results demonstrate that we can switch on or off the surface layer of DN gels by either substrate chemistry or compression during the $2^{\text {nd }}$ network polymerization.

We further analyzed the surface structure and modulus, in comparison with the bulk quantities for DN/Glass, DN/Si-PET and DN/Glass-C hydrogels synthesized by different formulations, and the results are summarized in Figure 2 and 3 . Figure 2A shows that the polymer surface density of SN PAMPS and 
SN PAAm hydrogels increases with the crosslinking degree $\left(X_{x_{1}}, X_{x_{2}}\right)$, due to the decrease of the volumetric swelling degree $(Q)$ at increased crosslinking degree. The polymer densities can be tuned in a range of $0.2 \sim 4.5 \mathrm{wt} \%$ and $4.2 \sim 15.2 \mathrm{wt} \%$ for PAMPS $\left(W_{M 1}\right)$ and PAAm $\left(W_{M 2}\right)$, respectively, by adjusting the crosslinking degree. Due to its polyelectrolyte nature, the swelling degree of PAMPS hydrogels is much higher than that of the neutral PAAm hydrogels even at a high crosslinking degree. The swelling degree of PAMPS in $2^{\text {nd }}$ network precursor solution is almost the same to that of PAMPS in water because the AAm monomer is neutral ${ }^{38}$.

The changes in surface polymer density and electric potential of DN gels in accordance to the $1^{\text {st }}$ network crosslinking degree $\left(X_{x_{1}}\right)$ is shown in Figure 2B. On DN hydrogels, the PAMPS surface densities $\left(W_{M 1}\right)$ between DN/Glass, DN/Si-PET and DN/Glass-C is different because of the presence or absence of the surface layer (upper left side). As the $1^{\text {st }}$ network crosslinking $\left(X_{X_{1}}\right)$ increases, the PAMPS surface densities of DN/Si-PET and DN/Glass-C hydrogels increased, in agreement with that of the SN PAMPS hydrogel. However, on the DN/Glass hydrogel surface, the PAMPS density is below a weight fraction of $1 \mathrm{wt} \%$ regardless of the change in the $X_{x 1}$ for the whole crosslinking range. The PAAm surface density on DN/Si-PET and DN/Glass-C hydrogels slightly increases with increasing PAMPS network crosslinking $\left(X_{X 1}\right)$, though the amount of crosslinker for the PAAm network for all DN hydrogels was kept constant ( $X_{X 2}: 0.1$ mol\%). However, on the DN/Glass hydrogel, the PAAm surface density is higher than that of the DN/Si-PET and DN/Glass-C hydrogels, showing almost a constant value, independent of the change in $X_{x 1}$. The increase in the PAAm density with $X_{x 1}$ on DN/Si-PET and DN/Glass-C gels is because the swelling of the PAAm is constrained by the presence of PAMPS. The higher the $X_{x 1}$ of the $1^{\text {st }}$ network, the more the PAAm network is constrained ${ }^{15}$. The surface molar ratio of PAMPS to PAAm $\left(C_{M 1} / C_{M 2}\right)$ increases with the increase in the $1^{\text {st }}$ network crosslinking degree for DN/Si-PET and DN/Glass-C gels while it keeps a low but constant value for DN/Glass gels (Figure 2B, lower left side). By cutting the surface hydrogel we exposed the bulk material so that a comparison between the surface and the bulk molar ratio $C_{M 1} / C_{M 2}$ is possible (Figure $2 \mathrm{~B}$, upper right side). While the bulk values were in good agreement with the theoretical values, the surface molar ratio $C_{M 1} / C_{M 2}$ is about 2 times higher than 
the bulk values. The theoretical values were estimated from, $C_{M 1} / C_{M 2}=Q^{-1} C_{M 1} / C_{M 2}$, where $C_{M 1}$ and $C_{M 2}$

355 are the feed monomer concentrations of the $1^{\text {st }}$ network and the $2^{\text {nd }}$ network, respectively, and $Q$ is

volumetric swelling degree of PAMPS hydrogels in AAm solution. The high molar ratio on the surface

compared to the bulk probably originates from the faster polymerization of the PAMPS hydrogels near the glass substrates in relation to the central region due to the higher UV intensity at the surface

regions. The increase in the amount of crosslinker $\left(X_{X_{1}}\right)$ reduces the swelling of the PAMPS hydrogel in the $2^{\text {nd }}$ network precursor solution and consequently increases the number of negative charges

through the PAMPS density. Therefore, the electric surface potential $\left(\Delta \Phi_{S}\right)$, defined as the initial

2B, lower right side). Similar increase in surface electric potential was published previously, when

AMPS because of a low conversion rate in the polymerization of highly crosslinked PAMPS.
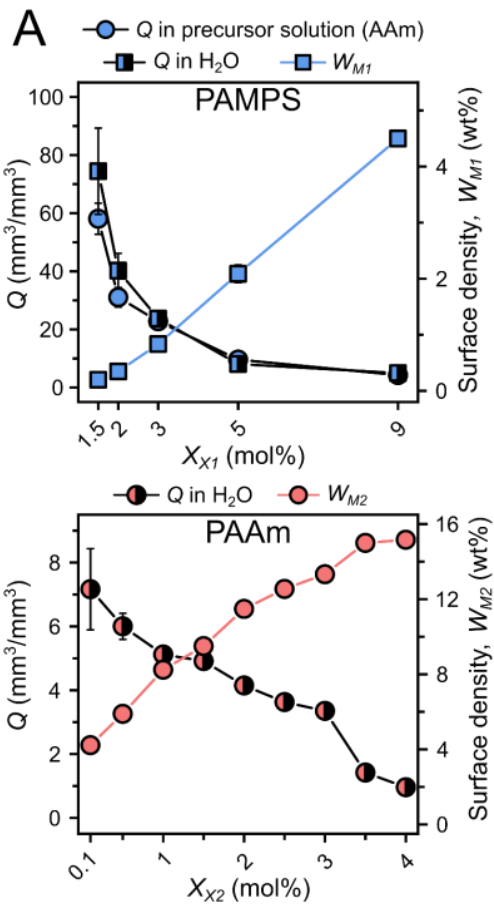

B
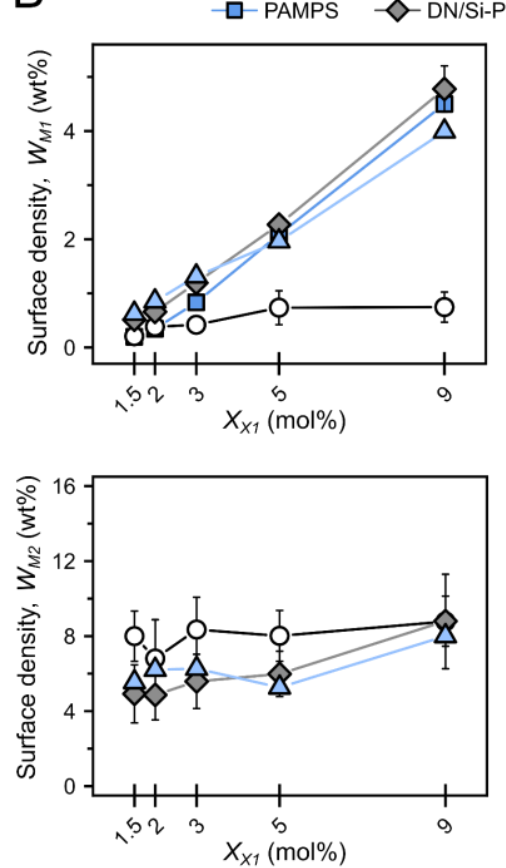

$\triangle$ DN/Glass-C -O-DN/Glass
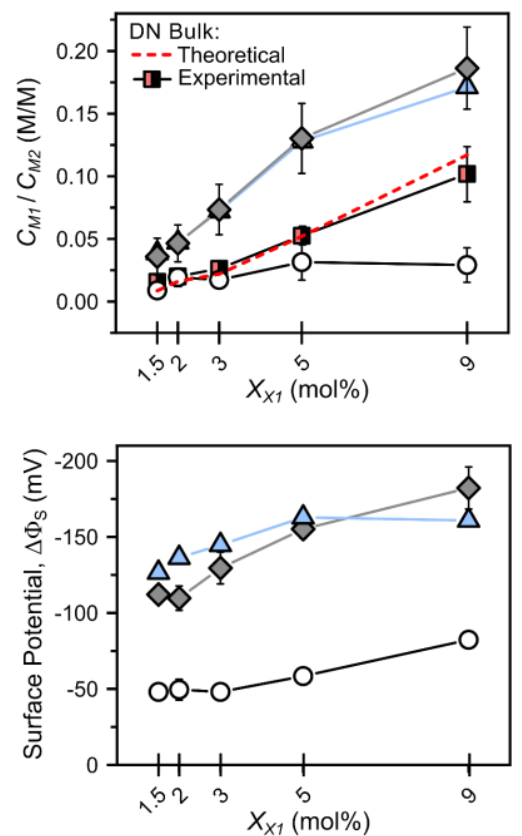

Figure 2: Regulation of polymer surface density $\left(W_{M 1}, W_{M 2}\right)$ by crosslinking degree $\left(X_{X 1}, X_{X 2}\right)$ of the

first network. (A) The volumetric swelling degree $(Q)$ and the corresponding surface polymer density $\left(W_{M 1}, W_{M 2}\right)$ of SN PAMPS and PAAm hydrogels (mean \pm SD, $n=3$ hydrogels per symbol) for varied crosslinking degree $\left(\mathrm{X}_{\mathrm{x}_{1}}, \mathrm{X}_{\mathrm{x} 2}\right)$. (B) DN hydrogel surface polymer density $\left(W_{M 1}, W_{\mathrm{M} 2}\right)$, the corresponding 
molar concentration ratio $\left(C_{M 1} / C_{M 2}\right)$, and the surface potential $\left(\Delta \Phi_{S}\right)$ for DN gels prepared with various

$3741^{\text {st }}$ network crosslinking degree $\left(X_{X_{1}}\right)$ (mean \pm SD, sample groups SN PAMPS, DN/Si-PET, DN/Glass contained sample sizes of $n=3-6$ hydrogels per symbol, and for DN/Glass-C $n=1$ hydrogel per symbol). The standard deviations (SD) of the PAAm density values tend to be high because the low accuracy of the ATR/FT-IR data used for the quantitative analysis resulted in a poorly fitted calibration curve (Figure S3, Figures S4).

As Figure 3A demonstrates, in the case of DN/Glass hydrogels the surface layer thickness is between 3 - $4 \mu \mathrm{m}$ for different $X_{x 1}$. Because of the presence of the surface layer, the elastic modulus at the surface is lower than the bulk in the case of the DN/Glass hydrogel, and only weakly increased with $X_{x 1}$. However, the bulk modulus of the DN/Glass hydrogel increases with increasing $1^{\text {st }}$ network crosslinking $\left(X_{x_{1}}\right)$, showing the same values as the bulk elastic modulus of DN/Si-PET hydrogels. For SN PAMPS and DN/Si-PET hydrogels, the surface and the bulk elastic modulus both increase with $X_{X 1}$.

From the above results, we can tune the bulk modulus while keeping the same surface chemistry. As shown in Figure 3B, when the bulk elastic modulus of DN/Glass and DN/Si-PET was changed through the $1^{\text {st }}$ network crosslinking degree, the polymer surface densities evolved differently, depending on the presence or absence of the surface layer. When no surface layer is present, the chemical structure at the surface is controlled through the PAMPS/PAAm ratio and the swelling in the $2^{\text {nd }}$ network precursor solution. However, when there is a surface layer, the surface chemical structure depends on the formulation of the $2^{\text {nd }}$ network precursor solution and is independent of the bulk elastic modulus. As shown for the SN PAAm hydrogels, polymer surface density and modulus increase with crosslinking density $X_{x_{2}}$. By varying $X_{x_{1}}$ and $X_{X_{2}}$ of the DN/Glass hydrogel, it is possible to individually tune the surface layer polymer density (or modulus) and the bulk polymer density (or modulus). 

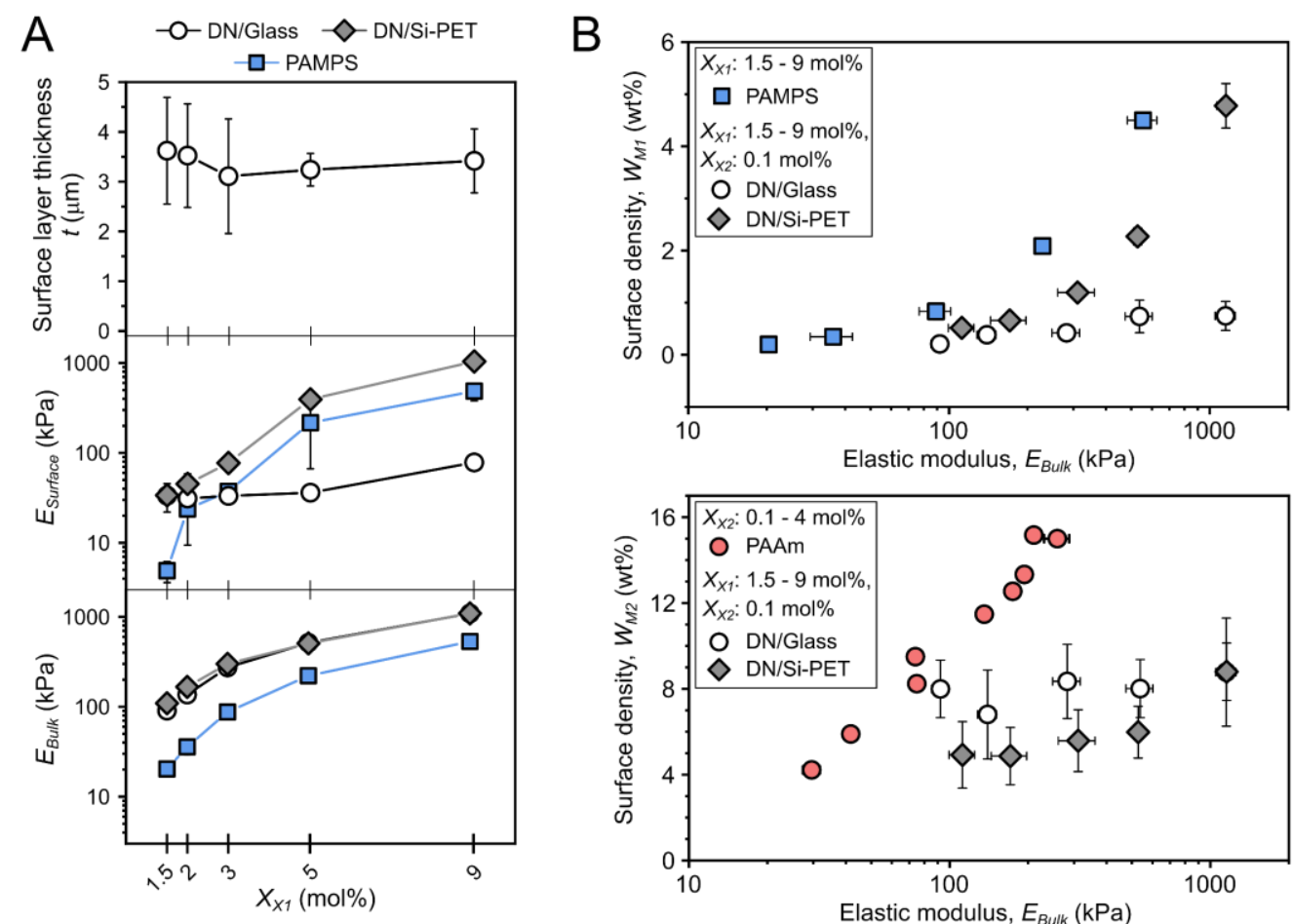

Figure 3: Effect of Surface layer thickness on elastic modulus. (A) The surface layer of $3-4 \mu \mathrm{m}$ thickness $(t)$ on the DN/Glass hydrogel affects the elastic modulus at the surface $\left(E_{\text {surface }}\right)$, (mean $\pm S D$, $\mathrm{n}=15-48$ technical replicates per symbol) but not the bulk ( $\left.E_{\text {Bulk }}\right)$. The sample groups SN PAMPS, SN PAAm, DN/Glass and DN/Si-PET contained sample sizes of $n=3-6$ hydrogels per symbol (mean \pm SD). (B) Independent tuning of bulk modulus and polymer surface densities $\left(W_{M 1}, W_{M 2}\right)$ by DN hydrogel synthesis.

\section{Control of the surface layer thickness}

To develop DN hydrogels with spatially modulated surface properties, the DN hydrogel was synthesized in a polymerization mold where the glass substrate was tilted which allows the regulation of the surface layer thickness, and through that, the control of the surface electric potential (Figure $4 A)$. During the $2^{\text {nd }}$ network synthesis, the $1^{\text {st }}$ network hydrogel was partially compressed on one side, through a spacer of height $h_{1}$, thinner than the hydrogel thickness $h$, to reach a maximum compression of $50 \mathrm{kPa}$ (Scheme S1). On the other side, a spacer of height $h_{2}$, thicker than the hydrogel was used, where the gap between the PAMPS network and the glass substrate was filled with excess AAm solution. The maximum gap distance creates a surface layer thickness of $0.9 \mathrm{~mm}\left(t \sim h_{2}-h_{1}\right)$ in the asprepared state. Because of the spacer height difference, a structural change at the surface-bulk transition zone through sample preparation should be induced. The cross-section view of the DN hydrogel shows that there is a clear difference in structure of the surface layer and the bulk region, as 
shown by the microscopic observations at position $8 \mathrm{~cm}$ (Figure 4A). The position is determined as the distance of the sampling position to the edge of the compressed hydrogel side. While the surface layer of $t \sim 800 \mu \mathrm{m}$ thick (Figure 4A, 1) shows a big porous structure, in the bulk material (Figure 4A, 3) a hierarchical structure of big pores filled with smaller ones is observed. The transition between the surface layer and the bulk is a clear cut (Figure 4A, 2, white dotted line), with a transition zone thinner than $20 \mu \mathrm{m}$. Even if there is a structural difference between the surface layer and the bulk observable, it must be noted that the observed porous structure is not resembling the native hydrogel structure because it is obtained through a freeze-drying process. The electric potential depth profiles measured at different positions reveal the gradual increase of the surface layer thickness with increasing distance to the compressed side of the DN hydrogel. Three typical depth profiles are shown in Figure 4B. The electric potential between the gel and the bath solution on the compressed side is above $-100 \mathrm{mV}$ (Distance: $0 \mathrm{~cm}$ ), related to the DN bulk potential. At positions of $2 \mathrm{~cm}$ and $6 \mathrm{~cm}$, the surface layer thickness increased so that the surface potential is weakened to a value of $-20 \mathrm{mV}$, related to the PAAm surface layer. The increase in surface thickness along the distance from the compressed side, analyzed by the electric potential depth profiles, is summarized in Figure 4C. On the compressed side (Position: $0 \mathrm{~cm}$ ), no surface layer is formed, and the surface potential of the gel is the same as the bulk, showing a value of $-120 \mathrm{mV}$. The surface potential abruptly changes from $-120 \mathrm{mV}$ to $-20 \mathrm{mV}$ between positions of 1 and $2 \mathrm{~cm}$, due to the formation of surface layer. With the increase of the distance to the compressed side, the surface layer thickness gradually increases and reaches values higher than $1 \mathrm{~mm}$ at the position of $9 \mathrm{~cm}$, while the surface potential remains constant at $-20 \mathrm{mV}$. Consequently, we obtained a hydrogel surface with a strong negatively charged region $\left(\Delta \Phi_{\mathrm{S}}=-120 \mathrm{mV}\right.$, blue $)$ that switches within $1 \mathrm{~cm}$ to an almost neutral $\left(\Delta \Phi_{s}=-20 \mathrm{mV}\right.$, red) region. Since the surface layer thickness increases with the distance from the compressed side, the surface modulus integrated from the surface layer and the bulk might change with the distance as well ${ }^{50}$. Such a surface might be used for studying the modulus effect on cells with a constant surface chemistry. 


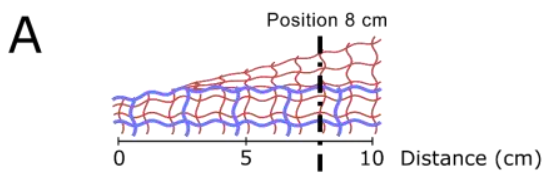

Position $8 \mathrm{~cm}$ : cross section view

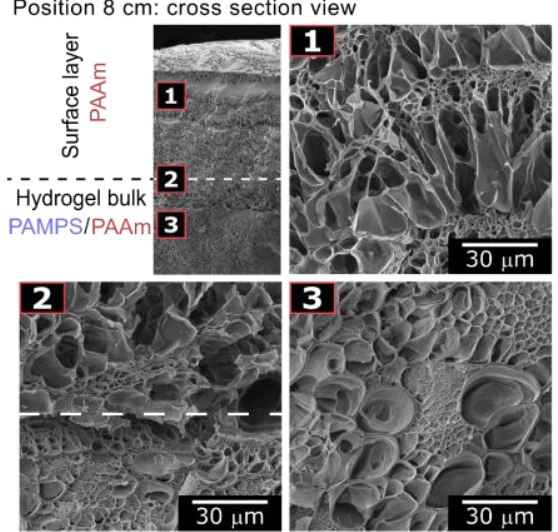

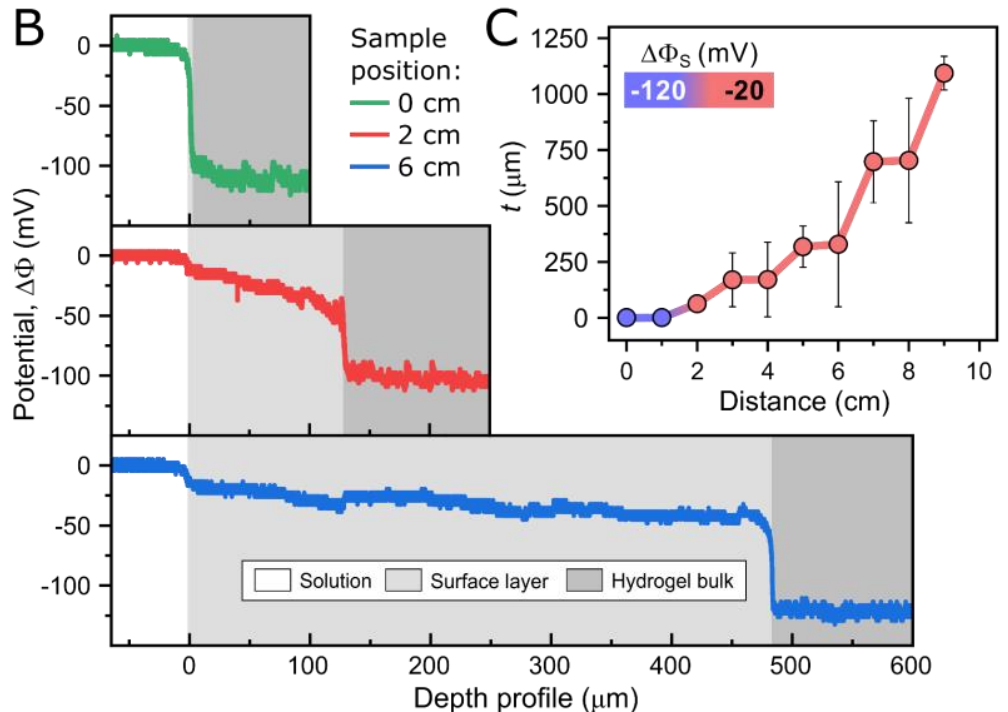

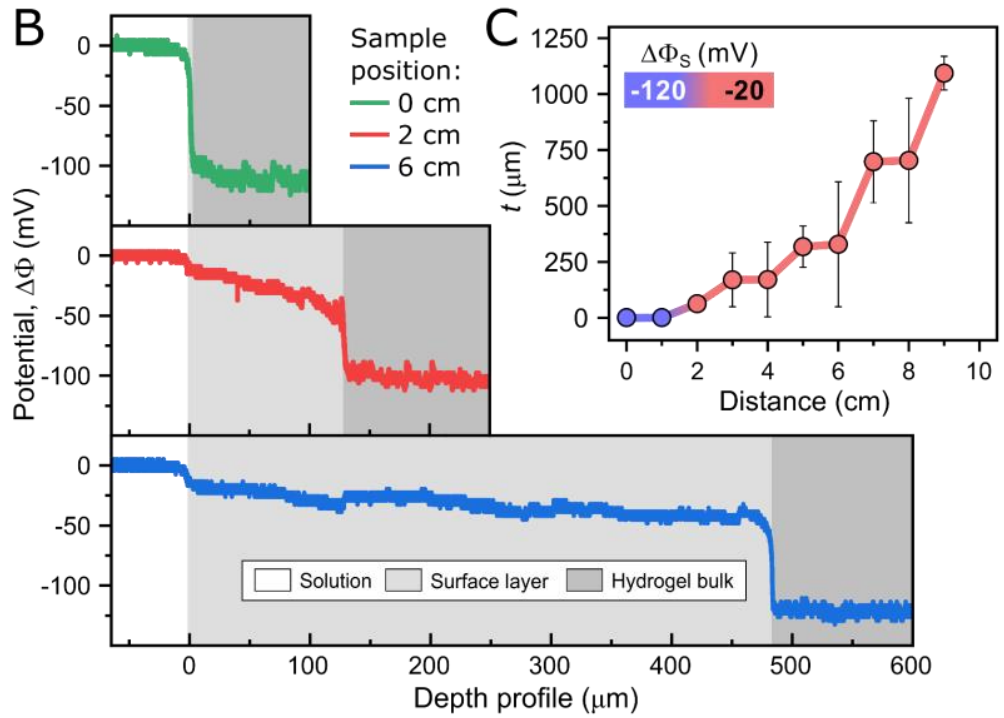

Figure 4: Control of PAAm layer thickness on the DN/Glass surface from micro-to-macro scale. (A) The $2^{\text {nd }}$ network polymerization setup where the spacer heights (Scheme S1) and the compressive stress regulate the surface layer thickness of the DN hydrogel (PAMPS/PAAm, 1-3-1/2-0.1-0.1). The DN hydrogel SEM cross-sectional view reveals a surface layer with thickness $t \sim 800 \mu \mathrm{m}$, with structural details on the PAAm surface layer (1), the transition zone $(2$, white dotted line represents the surfacebulk transition) and the DN bulk structure (3). (B) Selected electric potential depth profiles of the DN hydrogel at specific positions showing the surface layer thickness gradient. (C) Position profile of surface layer thickness and the corresponding surface potential $\left(\Delta \Phi_{s}\right)$ visualized by a blue (strong charged) and red (weak charged) color map ( $n=2-5$ measurements per position).

\section{Cell adhesion to DN hydrogels with varied surface potential}

The independent tuning of the surface and bulk properties of DN hydrogels by the surface-bulk structure transition allows us to control the surface potential of DN gels. Here, we investigate how the surface potentials of the hydrogels influence the cell attachment. As an example, mesenchymal stem cell attachment on various types of synthesized DN hydrogels was monitored in a cell culture experiment.

Figure 5 shows the morphology of the mesenchymal stem cells after 3 days of incubation on various hydrogels with different surface potentials. When the hydrogel surface potential was weaker than -60 $\mathrm{mV}$, as seen on PAAm and DN/Glass hydrogels, cells did not adhere to the surface, instead they aggregated to multicellular spheroids. On hydrogels with surface potential stronger than $-100 \mathrm{mV}$, as seen on PAMPS, DN/Glass-C, and DN/Si-PET hydrogels, cells started to adhere. On the SN PAMPS hydrogel the individual cells were circularly spread, while on the DN/Glass-C and DN/Si-PET hydrogels 
DN/Si-PET hydrogels, on the low crosslinked sample ( $X_{X 1} 1.5$ mol\%) dome-shaped adherent cell

aggregates were observed, but as the crosslinking degree increased the cells gradually become slightly

more spread and the dome-shaped aggregates were not observable anymore. The cell-substrate
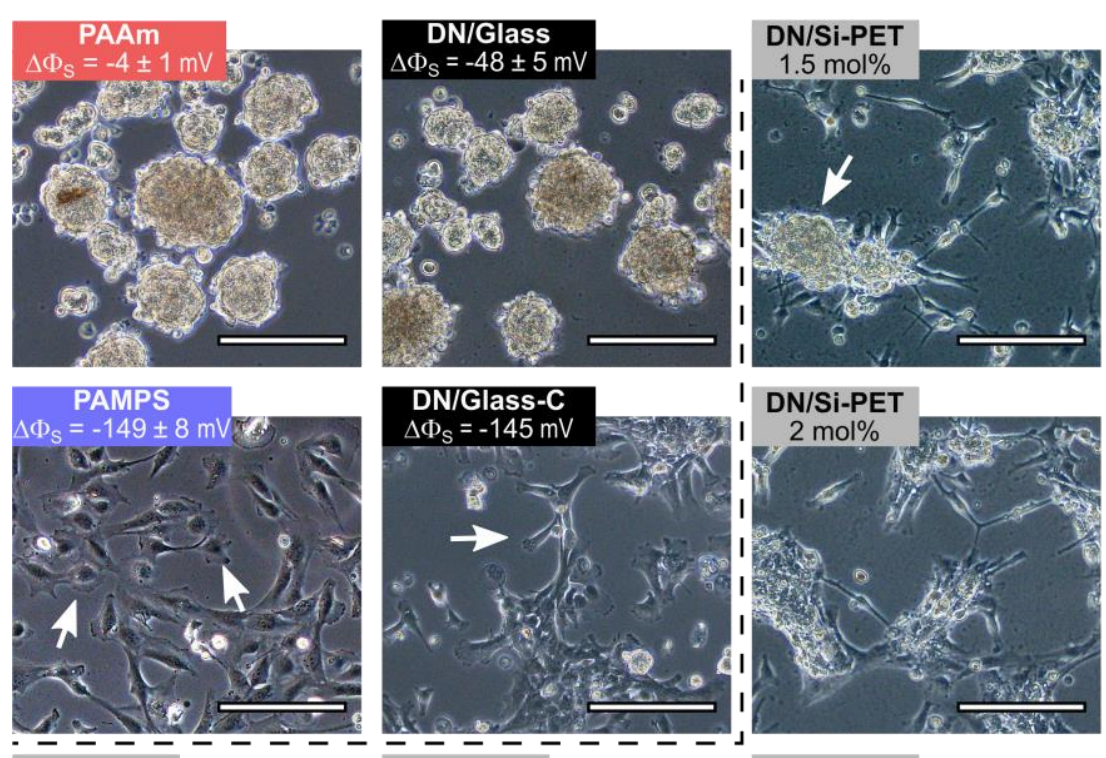

I DN/Si-PET
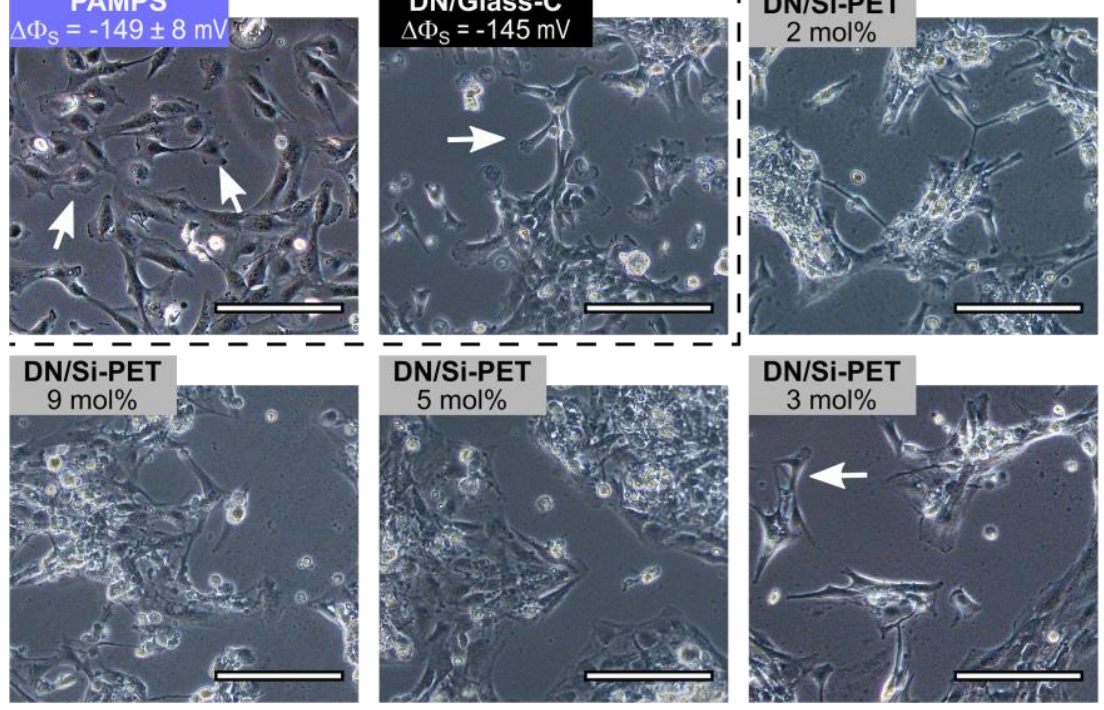

Figure 5: Increasing hydrogel surface potential induces cell attachment. Phase-contrast microscopic images (10 x magnification) of mesenchymal stem cells cultured on hydrogels for 3 days. Low surface electric potential facilitates stem cell aggregation (SN PAAm and DN/Glass) while high surface electric potential results in cell-hydrogel adhesion (SN PAMPS, DN/Glass-C, and DN/Si-PET). The scale bars represent $180 \mu \mathrm{m}$. The surface electric potentials for the DN/Si-PET hydrogels are shown in Figure 2B. 


\section{Summary and Conclusion}

477 The surface energy and charge of substrates used in $2^{\text {nd }}$ network synthesis play a crucial role in 478 determining DN hydrogel surface chemistry and surface stiffness. While the use of a low energy 479 molding substrate facilitates the synthesis of DN hydrogels with both networks present at the surface, 480 the electrostatic interactions between glass and like-charged polyelectrolyte hydrogels are responsible 481 for the formation of a $2^{\text {nd }}$ network polymer surface layer. The regulation of the DN hydrogel surface potential is mainly related to the $2^{\text {nd }}$ network surface layer thickness that is modifiable through molding substrate surface energy or normal compression during synthesis. If no surface layer is present, a secondary regulation of the surface potential is possible via the molar ratio between the polyelectrolyte and neutral polymer. Usually, DN hydrogels made from two chemically crosslinked networks are synthesized in a two-step approach, which is time consuming and work intensive. However, with the here-proposed modifications, the second synthesis step is an easily accessible technique that fabricates not just a tough hydrogel but simultaneously functionalizes the hydrogel surface, which will be useful in controllable surface friction, cell scaffolding, and in vivo applications.

The combination of the ATR/FT-IR and the microelectrode technique has proven to be a powerful approach for characterizing hydrogel surfaces since each technique makes up for the disadvantage of the other technique. The microelectrode technique, which is highly sensitive in detecting electric potentials but has a poor spatial resolution in the depth profile, complements the ATR/FT-IR technique, which gives structural information on the topmost hydrogel layer with lower sensitivity.

The cell experiment proves that the previously observed phenomena, where cells do not adhere to DN hydrogels, is not valid for DN hydrogels in general, but is a consequence of the synthesis condition. Above a critical value, the surface PAMPS/PAAm ratios, applying to a wide range of DN hydrogel compositions, have just a minor impact on the surface electric potential and the cell morphology. This makes clear that publications investigating the interactions between cells and DN hydrogels must explicitly describe the utilized polymerization substrate and the related surface potentials, since it 
drastically changes the hydrogels cell-adhesive properties. Recently, there is evidence that the induced stem cell state, of interest to cell therapies, is enhanced by cell aggregation. Therefore, the detailed hydrogel characterization and physicochemical decoupling based on the regulation of the surface-bulk transition described here could be applied to investigation how the morphological stem cell changes alter gene expression. Regarding such studies, additional evaluation of hydrogel-protein adsorption and cell viabilities on the synthesized hydrogels is necessary in the future.

\section{Acknowledgements}

This research was supported by Grant-in-Aid for Scientific Research (S) (No. 17H06144). The Institute for Chemical Reaction Design and Discovery (ICReDD) was established by the World Premier International Research Initiative (WPI), MEXT, Japan. M. Frauenlob thanks MEXT, Japan for the provided scholarship to conduct his PhD study, A. Giustiniani as well as D. Schenz for the constructive discussions regarding figure design and Y. Z. Guo and T. Sedlacik for the experimental support.

\section{Supporting information}

The supporting information contains Scheme S1, Table S1 and Figure S1 - 5 related to this article.

\section{References}

(1) Matsuda, T.; Kawakami, R.; Namba, R.; Nakajima, T.; Gong, J. P. Mechanoresponsive SelfGrowing Hydrogels Inspired by Muscle Training. Science 2019, 363 (6426), 504-508. https://doi.org/10.1126/science.aau9533.

(2) Liu, S.; Li, L. Ultrastretchable and Self-Healing Double-Network Hydrogel for 3D Printing and Strain Sensor. ACS Appl. Mater. Interfaces 2017, 9 (31), 26429-26437. https://doi.org/10.1021/acsami.7b07445.

(3) King, D. R.; Sun, T. L.; Huang, Y.; Kurokawa, T.; Nonoyama, T.; Crosby, A. J.; Gong, J. P. Extremely Tough Composites from Fabric Reinforced Polyampholyte Hydrogels. Mater. Horizons 2015, 2 (6), 584-591. https://doi.org/10.1039/C5MH00127G.

(4) Nonoyama, T.; Wada, S.; Kiyama, R.; Kitamura, N.; Mredha, M. T. I.; Zhang, X.; Kurokawa, T.; 
Nakajima, T.; Takagi, Y.; Yasuda, K.; et al. Double-Network Hydrogels Strongly Bondable to Bones by Spontaneous Osteogenesis Penetration. Adv. Mater. 2016, 28 (31), 6740-6745. https://doi.org/10.1002/adma.201601030.

(5) Yan, Y.; Li, M.; Yang, D.; Wang, Q.; Liang, F.; Qu, X.; Qiu, D.; Yang, Z. Construction of Injectable Double-Network Hydrogels for Cell Delivery. Biomacromolecules 2017, 18 (7), 2128-2138. https://doi.org/10.1021/acs.biomac.7b00452.

(6) Kiyama, R.; Nonoyama, T.; Wada, S.; Semba, S.; Kitamura, N.; Nakajima, T.; Kurokawa, T.; Yasuda, K.; Tanaka, S.; Gong, J. P. Micro Patterning of Hydroxyapatite by Soft Lithography on Hydrogels for Selective Osteoconduction. Acta Biomater. 2018, 81, 60-69. https://doi.org/10.1016/j.actbio.2018.10.002.

(7) Zhao, Y.; Nakajima, T.; Yang, J. J.; Kurokawa, T.; Liu, J.; Lu, J.; Mizumoto, S.; Sugahara, K.; Kitamura, N.; Yasuda, K.; et al. Proteoglycans and Glycosaminoglycans Improve Toughness of Biocompatible Double Network Hydrogels. Adv. Mater. 2014, 26 (3), 436-442. https://doi.org/10.1002/adma.201303387.

(8) Ajiro, H.; Watanabe, J.; Akashi, M. Cell Adhesion and Proliferation on Poly(N-Vinylacetamide) Hydrogels and Double Network Approaches for Changing Cellular Affinities. Biomacromolecules 2008, 9 (2), 426-430. https://doi.org/10.1021/bm701221c.

(9) Higa, K.; Kitamura, N.; Kurokawa, T.; Goto, K.; Wada, S.; Nonoyama, T.; Kanaya, F.; Sugahara, K.; Gong, J. P.; Yasuda, K. Fundamental Biomaterial Properties of Tough GlycosaminoglycanContaining Double Network Hydrogels Newly Developed Using the Molecular Stent Method. Acta Biomater. 2016, 43, 38-49. https://doi.org/10.1016/j.actbio.2016.07.023.

(10) Wada, S.; Kitamura, N.; Nonoyama, T.; Kiyama, R.; Kurokawa, T.; Gong, J. P.; Yasuda, K. Hydroxyapatite-Coated Double Network Hydrogel Directly Bondable to the Bone: Biological and Biomechanical Evaluations of the Bonding Property in an Osteochondral Defect. Acta Biomater. 2016, 44, 125-134. https://doi.org/10.1016/j.actbio.2016.08.016.

(11) Ye, Y. N.; Frauenlob, M.; Wang, L.; Tsuda, M.; Sun, T. L.; Cui, K.; Takahashi, R.; Zhang, H. J.; Nakajima, T.; Nonoyama, T.; et al. Tough and Self-Recoverable Thin Hydrogel Membranes for Biological Applications. Adv. Funct. Mater. 2018, 28 (31), 1801489. https://doi.org/10.1002/adfm.201801489.

(12) Yin, H.; Akasaki, T.; Lin Sun, T.; Nakajima, T.; Kurokawa, T.; Nonoyama, T.; Taira, T.; Saruwatari, Y.; Ping Gong, J. Double Network Hydrogels from Polyzwitterions: High Mechanical Strength and Excellent Anti-Biofouling Properties. J. Mater. Chem. B 2013, 1 (30), 3685-3693. 
https://doi.org/10.1039/С3TB20324G.

559

560

561

562

563

564

565

566

567

568

569

570

571

572

573

574

575

576

577

578

579

580

581

582

583

584

585

586

(13) Gong, J. P. Why Are Double Network Hydrogels so Tough? Soft Matter 2010, 6 (12), 2583. https://doi.org/10.1039/b924290b.

(14) Nakajima, T.; Furukawa, H.; Tanaka, Y.; Kurokawa, T.; Osada, Y.; Gong, J. P. True Chemical Structure of Double Network Hydrogels. Macromolecules 2009, 42 (6), 2184-2189. https://doi.org/10.1021/ma802148p.

(15) Ahmed, S.; Nakajima, T.; Kurokawa, T.; Anamul Haque, M.; Gong, J. P. Brittle-Ductile Transition of Double Network Hydrogels: Mechanical Balance of Two Networks as the Key Factor. Polymer (Guildf). 2014, 55 (3), 914-923. https://doi.org/10.1016/j.polymer.2013.12.066.

(16) Sun, J.-Y.; Zhao, X.; Illeperuma, W. R. K.; Chaudhuri, O.; Oh, K. H.; Mooney, D. J.; Vlassak, J. J.; Suo, Z. Highly Stretchable and Tough Hydrogels. Nature 2012, 489 (7414), 133-136. https://doi.org/10.1038/nature11409.

(17) Kwon, H. J.; Yasuda, K.; Ohmiya, Y.; Honma, K. ichi; Chen, Y. M.; Gong, J. P. In Vitro Differentiation of Chondrogenic ATDC5 Cells Is Enhanced by Culturing on Synthetic Hydrogels with Various Charge Densities. Acta Biomater. 2010, 6 (2), 494-501. https://doi.org/10.1016/j.actbio.2009.07.033.

(18) Chen, Y. M.; Tanaka, M.; Gong, J. P.; Yasuda, K.; Yamamoto, S.; Shimomura, M.; Osada, Y. Platelet Adhesion to Human Umbilical Vein Endothelial Cells Cultured on Anionic Hydrogel Scaffolds. Biomaterials 2007, 28 (10), 1752-1760. https://doi.org/10.1016/j.biomaterials.2006.12.005.

(19) Chen, Y. M.; Shiraishi, N.; Satokawa, H.; Kakugo, A.; Narita, T.; Gong, J. P.; Osada, Y.; Yamamoto, K.; Ando, J. Cultivation of Endothelial Cells on Adhesive Protein-Free Synthetic Polymer Gels. Biomaterials 2005, 26 (22), 4588-4596. https://doi.org/10.1016/j.biomaterials.2004.11.025.

(20) Inagaki, Y.; Kitamura, N.; Kurokawa, T.; Tanaka, Y.; Gong, J. P.; Yasuda, K.; Tohyama, H. Effects of Culture on PAMPS/PDMAAm Double-Network Gel on Chondrogenic Differentiation of Mouse C3H10T1/2 Cells: In Vitro Experimental Study. BMC Musculoskelet. Disord. 2014, 15 (1), 320. https://doi.org/10.1186/1471-2474-15-320.

(21) Kitamura, N.; Kurokawa, T.; Fukui, T.; Gong, J. P.; Yasuda, K. Hyaluronic Acid Enhances the Effect of the PAMPS/PDMAAm Double-Network Hydrogel on Chondrogenic Differentiation of ATDC5 Cells. BMC Musculoskelet. Disord. 2014, 15 (1), 222. https://doi.org/10.1186/1471- 
591

592

593

594

595

596

597

598

599

600

601

602

603

604

605

606

607

608

609

610

611

612

613

614

615

616

617

618

619

620

(22) Maeda, E.; Tsutsumi, T.; Kitamura, N.; Kurokawa, T.; Ping Gong, J.; Yasuda, K.; Ohashi, T. Significant Increase in Young's Modulus of ATDC5 Cells during Chondrogenic Differentiation Induced by PAMPS/PDMAAm Double-Network Gel: Comparison with Induction by Insulin. J. Biomech. 2014, 47 (13), 3408-3414. https://doi.org/10.1016/j.jbiomech.2014.07.021.

(23) Yasuda, K.; Kitamura, N.; Gong, J. P.; Arakaki, K.; Kwon, H. J.; Onodera, S.; Chen, Y. M.; Kurokawa, T.; Kanaya, F.; Ohmiya, Y.; et al. A Novel Double-Network Hydrogel Induces Spontaneous Articular Cartilage Regeneration in Vivo in a Large Osteochondral Defect. Macromol. Biosci. 2009, 9 (4), 307-316. https://doi.org/10.1002/mabi.200800223.

(24) Higa, K.; Kitamura, N.; Goto, K.; Kurokawa, T.; Gong, J. P.; Kanaya, F.; Yasuda, K. Effects of Osteochondral Defect Size on Cartilage Regeneration Using a Double-Network Hydrogel. BMC Musculoskelet. Disord. 2017, 18 (1), 210. https://doi.org/10.1186/s12891-017-1578-1.

(25) Engler, A. J.; Sen, S.; Sweeney, H. L.; Discher, D. E. Matrix Elasticity Directs Stem Cell Lineage Specification. Cell 2006, 126 (4), 677-689. https://doi.org/10.1016/j.cell.2006.06.044.

(26) Das, R. K.; Gocheva, V.; Hammink, R.; Zouani, O. F.; Rowan, A. E. Stress-Stiffening-Mediated Stem-Cell Commitment Switch in Soft Responsive Hydrogels. Nat. Mater. 2016, 15 (3), 318325. https://doi.org/10.1038/nmat4483.

(27) Riedl, A.; Schlederer, M.; Pudelko, K.; Stadler, M.; Walter, S.; Unterleuthner, D.; Unger, C.; Kramer, N.; Hengstschläger, M.; Kenner, L.; et al. Comparison of Cancer Cells in 2D vs 3D Culture Reveals Differences in AKT-MTOR-S6K Signaling and Drug Responses. J. Cell Sci. 2017, 130 (1), 203-218. https://doi.org/10.1242/jcs.188102.

(28) Wang, W.; Itaka, K.; Ohba, S.; Nishiyama, N.; Chung, U.; Yamasaki, Y.; Kataoka, K. 3D Spheroid Culture System on Micropatterned Substrates for Improved Differentiation Efficiency of Multipotent Mesenchymal Stem Cells. Biomaterials 2009, 30 (14), 2705-2715. https://doi.org/10.1016/j.biomaterials.2009.01.030.

(29) Schneider, G. B.; English, A.; Abraham, M.; Zaharias, R.; Stanford, C.; Keller, J. The Effect of Hydrogel Charge Density on Cell Attachment. Biomaterials 2004, 25 (15), 3023-3028. https://doi.org/10.1016/j.biomaterials.2003.09.084.

(30) Han, L.; Mao, Z.; Wu, J.; Zhang, Y.; Gao, C. Influences of Surface Chemistry and Swelling of Salt-Treated Polyelectrolyte Multilayers on Migration of Smooth Muscle Cells. J. R. Soc. Interface 2012, 9 (77), 3455-3468. https://doi.org/10.1098/rsif.2012.0546. 
(31) Lampin, M.; Warocquier-Clerout, R.; Legris, C.; Degrange, M.; Sigot-Luizard, M. F. Correlation between Substratum Roughness and Wettability, Cell Adhesion, and Cell Migration. J. Biomed. Mater. Res. 1997, 36 (1), 99-108. https://doi.org/10.1002/(SICI)10974636(199707)36:1<99::AID-JBM12>3.0.CO;2-E.

(32) Charras, G.; Sahai, E. Physical Influences of the Extracellular Environment on Cell Migration. Nat. Rev. Mol. Cell Biol. 2014, 15 (12), 813-824. https://doi.org/10.1038/nrm3897.

(33) Chen, Y. M.; Gong, J. P.; Tanaka, M.; Yasuda, K.; Yamamoto, S.; Shimomura, M.; Osada, Y. Tuning of Cell Proliferation on Tough Gels by Critical Charge Effect. J. Biomed. Mater. Res. Part A 2009, 88A (1), 74-83. https://doi.org/10.1002/jbm.a.31869.

(34) Tan, F.; Liu, J.; Liu, M.; Wang, J. Charge Density Is More Important than Charge Polarity in Enhancing Osteoblast-like Cell Attachment on Poly(Ethylene Glycol)-Diacrylate Hydrogel. Mater. Sci. Eng. C 2017, 76, 330-339. https://doi.org/10.1016/j.msec.2017.03.051.

(35) Chang, H.-Y.; Kao, W.-L.; You, Y.-W.; Chu, Y.-H.; Chu, K.-J.; Chen, P.-J.; Wu, C.-Y.; Lee, Y.-H.; Shyue, J.-J. Effect of Surface Potential on Epithelial Cell Adhesion, Proliferation and Morphology. Colloids Surfaces B Biointerfaces 2016, 141, 179-186. https://doi.org/10.1016/j.colsurfb.2016.01.049.

(36) Gong, J. P.; Kagata, G.; Osada, Y. Friction of Gels. 4. Friction on Charged Gels. J. Phys. Chem. B 1999, 103 (29), 6007-6014. https://doi.org/10.1021/jp990256v.

(37) Volkov, A. G.; Markin, V. S. Electric Properties of Oil/Water Interfaces. 2004, 4, 91-182. https://doi.org/10.1016/S1573-4285(04)80006-1.

(38) Polyelectrolyte Gels; Harland, R. S., Prud'homme, R. K., Eds.; ACS Symposium Series; American Chemical Society: Washington, DC, 1992. https://doi.org/10.1021/bk-1992-0480.

(39) Gong, P.; Genzer, J.; Szleifer, I. Phase Behavior and Charge Regulation of Weak Polyelectrolyte Grafted Layers. Phys. Rev. Lett. 2007, 98 (1), 018302. https://doi.org/10.1103/PhysRevLett.98.018302.

(40) Erbaş, A.; Olvera de la Cruz, M. Interactions between Polyelectrolyte Gel Surfaces. Macromolecules 2016, 49 (23), 9026-9034. https://doi.org/10.1021/acs.macromol.6b01416.

(41) Sokoloff, J. B. Theory of Hydrostatic Lubrication for Two Like-Charge Polymer Hydrogel Coated Surfaces. Soft Matter 2010, 6 (16), 3856. https://doi.org/10.1039/c000252f.

(42) Gong, J. P. Friction and Lubrication of Hydrogels-Its Richness and Complexity. Soft Matter 2006, 2 (7), 544-552. https://doi.org/10.1039/B603209P. 
(43) Maréchal, Y. IR Spectroscopy of an Exceptional H-Bonded Liquid: Water. J. Mol. Struct. 1994, 322 (C), 105-111. https://doi.org/10.1016/0022-2860(94)87025-X.

(44) Yezek, L. P.; van Leeuwen, H. P. An Electrokinetic Characterization of Low Charge Density Cross-Linked Polyacrylamide Gels. J. Colloid Interface Sci. 2004, 278 (1), 243-250. https://doi.org/10.1016/j.jcis.2004.05.026.

(45) Guo, H.; Kurokawa, T.; Takahata, M.; Hong, W.; Katsuyama, Y.; Luo, F.; Ahmed, J.; Nakajima, T.; Nonoyama, T.; Gong, J. P. Quantitative Observation of Electric Potential Distribution of Brittle Polyelectrolyte Hydrogels Using Microelectrode Technique. Macromolecules 2016, 49 (8), 3100-3108. https://doi.org/10.1021/acs.macromol.6b00037.

(46) Gong, J. P.; Katsuyama, Y.; Kurokawa, T.; Osada, Y. Double-Network Hydrogels with Extremely High Mechanical Strength. Adv. Mater. 2003, 15 (14), 1155-1158. https://doi.org/10.1002/adma.200304907.

(47) Furniss, B. S.; Hannaford, A. J.; Smith, P. W. G.; Tatchell, A. R. Vogel's Textbook of Practical Organic Chemistry, 5th ed.; Harlow: Longman, 1989.

(48) Hertz, H. On the Contact of Elastic Solids. J. Reine Angew. Math. 1881, 92, 156-171.

(49) Eisenberg, D. S.; Crothers, D. M. Physical Chemistry: With Applications to the Life Sciences; Benjamin/Cummings Publishing Company Inc.: San Francisco, 1979.

(50) Hadden, W. J.; Young, J. L.; Holle, A. W.; McFetridge, M. L.; Kim, D. Y.; Wijesinghe, P.; TaylorWeiner, H.; Wen, J. H.; Lee, A. R.; Bieback, K.; et al. Stem Cell Migration and Mechanotransduction on Linear Stiffness Gradient Hydrogels. Proc. Natl. Acad. Sci. 2017, 114 (22), 5647-5652. https://doi.org/10.1073/pnas.1618239114.

(51) Arias, C. J.; Surmaitis, R. L.; Schlenoff, J. B. Cell Adhesion and Proliferation on the "Living" Surface of a Polyelectrolyte Multilayer. Langmuir 2016, 32 (21), 5412-5421. https://doi.org/10.1021/acs.langmuir.6b00784.

(52) Arias, C. J.; Keller, T. C. S.; Schlenoff, J. B. Quasi-Spherical Cell Clusters Induced by a Polyelectrolyte Multilayer. Langmuir 2015, 31 (23), 6436-6446. https://doi.org/10.1021/acs.langmuir.5b00678.

(53) Razafiarison, T.; Holenstein, C. N.; Stauber, T.; Jovic, M.; Vertudes, E.; Loparic, M.; Kawecki, M.; Bernard, L.; Silvan, U.; Snedeker, J. G. Biomaterial Surface Energy-Driven Ligand Assembly Strongly Regulates Stem Cell Mechanosensitivity and Fate on Very Soft Substrates. Proc. Natl. Acad. Sci. 2018, 115 (18), 4631-4636. https://doi.org/10.1073/pnas.1704543115. 